\title{
THE EFFECT OF CHANGES IN TRAFFIC FLOW ON MAMMAL ROAD KILL COUNTS
}

\author{
PAUL W. BRIGHT ${ }^{1}$ - ZOE BALMFORTH - JENNY L. MACPHERSON* \\ ${ }^{1}$ Royal Holloway University of London \\ Egham Hill, Egham, Surrey TW20 OEX, UK. \\ (phone: +44(0)1784-443772; fax: +44(0)1784-434326) \\ *Corresponding author \\ e-mail: jenny.macpherson@vwt.org.uk \\ (Received 29 $9^{\text {th }}$ Apr 2013; accepted $14^{\text {th }}$ Aug 2014)
}

\begin{abstract}
It is estimated that one million wild animals are killed on UK roads each year and these casualties are potentially a valuable source of data relating to the size and distribution of populations. Road kill surveys have the potential to form the basis of a nationwide mammal monitoring scheme with multi-species coverage and excellent area sampling. However it has been argued that road casualty counts cannot be used to monitor population change because variation in the number of road deaths is related more to variation in traffic flow than to population density. We used rabbit and hedgehog as study species to examine if there was a relationship between traffic flow and road kill counts. We found no correlation between changes in traffic flow and road counts of hedgehog or rabbit. We conclude that a decrease in road kill counts of these species is highly likely to represent a decrease in absolute abundance and would therefore be a good index with which to monitor population decline.
\end{abstract}

Keywords: traffic mortality, road kill, mammal monitoring

\section{Introduction}

It is estimated that one million animals are killed annually on UK roads (Slater, 1994), and these casualties represent a potentially very valuable source of data relating to the size and distribution of populations. Important advantages of using road casualty data to monitor mammals include the fact that road-kill surveys can simultaneously collect data on multiple species across broad geographic areas. While it would be impossible to design a single index of abundance or monitoring scheme capable of assessing numbers of all UK mammal species simultaneously (since some have very specific habits and/or locations), the broader the species range and area covered the better in terms of cost, efficiency and comparability. Furthermore, the fact that the most common mammalian casualties belong to easily identifiable species such as rabbit, Oryctolagus cuniculus, and hedgehog, Erinaceus europaeus, (Davies, 1957) means that there is no need for skilled experts to conduct surveys. Roads effectively form linear sampling devices across large areas of countryside, they are freely accessible to the public, and they are unlikely to suffer from problems associated with changing land use during or between surveys.

One of the most frequently voiced arguments against the use of casualty counts to monitor population change is that variation in the number of road deaths is related more to variation in traffic flow than to population density. Indeed, some studies have suggested that traffic flow is the single most important explanatory variable when considering variation in road casualty figures for certain taxa (Driessen et al., 1996; Fahrig et al., 1995). However, there are also several examples of studies suggesting that, although traffic flow has a role to play, it is less important than population density (e.g.

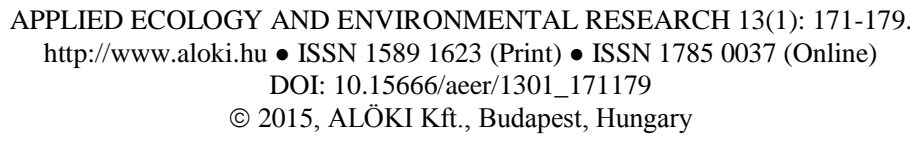


Morris and Morris, 1988; Brockie et al, 2009). Between 2001 and 2004 traffic flow in Great Britain increased by an estimated $5.09 \%$, or $1.69 \%$ per year (DTLR, 2005). Increased traffic flow could, irrespective of mammal population density, lead to an increase in mammal road counts, if the probability of a species crossing roads remains constant (or increases) but the probability of death while crossing roads increases in relation to traffic flow. Alternatively it could result in a decrease in mammal road kill counts, if a species is more inhibited from crossing roads as a result of greater traffic flow, or if road traffic mortality per se is having a biologically significant impact on a species population. A third possibility is that there would be no change in mammal road kill counts, if the increase in traffic flow is too small to influence road crossing behaviour or mortality. Of crucial importance may be whether traffic flow at times when mammals are active has increased sufficiently to influence road counts. Therefore, it is important to clarify the relationship between traffic flow and road casualty counts in order to determine the reliability of the latter as an index of mammal abundance.

A number of studies have included attempts to calibrate road casualty counts to estimates of abundance (Inbar and Mayer, 1999; Jahn, 1959; Mallick et al., 1998; McCaffery, 1973; Rolley and Lehman, 1992). Case (1978) showed that annual road-kill counts for pheasants (Phasianus colchicus) in Nebraska correlated well with a sightings survey carried out by rural postal delivery workers, and suggested that road casualty data may be a substitute or supplement to this census technique. The Illinois Department of Conservation compared racoon (Procyon lotor) road casualty numbers to spotlight surveys along roads and found a strong correlation, suggesting that numbers of road casualties recorded were generally accurate reflections of the numbers living in the surrounding area (Hicks, 1993). Studies of white-tailed deer populations have also shown correlations between spotlight surveys (Bellis and Graves, 1971), buck harvests (Arnold, 1978; McCaffery, 1973) and road casualties. In the UK, George et al (2011) found a direct relationship between numbers of rabbit road casualties and numbers living in the wider landscape.

Studies such as these reinforce the idea that road casualty figures could be accurately and reliably used in the UK to monitor mammal populations. Road-kill surveys have the potential to form the basis of a nationwide mammal monitoring scheme with multispecies coverage and excellent area sampling. Furthermore, the fact that unskilled volunteers could be employed as surveyors means that the necessary project effort and cost would be minimal.

\section{Methods}

Between August and September 2001 to 2004, 105 road transects were surveyed by passengers travelling by car. There were 15 transects in each of the seven landclass groups classified by the Centre for Ecology and Hydrology (Bunce et al., 1996). Each transect was approximately $97 \mathrm{~km}$ (60 miles) in length and included only singlecarriageway, non-urban roads (i.e., only those that did not pass through $\geq 3.2 \mathrm{~km}$ of built up land). Each covered 'A', 'B' and 'Minor' roads in the ratio 4:3:1, respectively. The nearest town, the odometer reading and the road number were recorded at the start and end of each transect, and at approximate $16 \mathrm{~km}$ intervals along each. In addition, at each of these points the National Grid Reference (NGR) was recorded (to the nearest 10m) using a Garmin global positioning system (GPS) receiver. 
Whilst travelling each transect, whenever the surveyor turned onto a new road the odometer reading and new road number were recorded, so that the route could be accurately plotted. Each individual road was classified as a segment of the respective transect. Traffic flow was recorded as the count of all oncoming vehicles encountered during a journey.

During each transect survey, every mammal road casualty encountered was noted and identified to species level (or recorded as unidentifiable if this was impossible). The time of each casualty sighting was recorded, along with the NGR of the casualty site.

As it was not possible to ensure that all transects were identical in length, counts of mammal road casualties were transformed into the number recorded per $100 \mathrm{~km}$ driven. The proportion of UK road area surveyed was calculated using data provided by the Department for Transport, Local Government and the Regions (DTLR; 2000).

Hedgehogs cross roads at a mean speed of $0.5 \mathrm{~m} / \mathrm{second}$ (S. Carter unpubl. data). We were unable to measure road crossing speeds of rabbits, but Garland (1983) suggests that their maximum running speed will be $15.5 \mathrm{~m} / \mathrm{second}$. Hedgehogs, under experimental conditions which could have reduced risk-aversion, perceived a threat from a vehicle, on average, when it was $70 \mathrm{~m}$ away. Rabbits, from the safety of the road verge, exhibited alarm responses to traffic that was, on average, $162 \mathrm{~m}$ from them (George, 2004). From these findings and the Department of Transport statistics referenced above, we can estimate how changes in traffic flow may have affected the probability that a road is crossed, the probability that a mammal once on a road will collide with a vehicle and thus the probability of mammal road traffic accidents. We assume a worse case scenario where mammals are crossing an A road $10 \mathrm{~m}$ wide, traffic is moving at $60 \mathrm{mph}(96 \mathrm{kph})$ and that hedgehogs cross roads during the night time period defined previously, whilst rabbits may cross at any time of the day or night. We assume that mammals do not cross roads when vehicles are within the distance that they show risk-aversion to traffic and that they cross roads at the speeds suggested above.

The traffic flow-dependent probability of road crossing $\left(P_{c}\right)$ is given by equation 1 .

$$
P_{c}=\frac{D V_{t} L_{t}}{L_{r}}
$$

$D$ is the distance at which a mammal detects approaching traffic from the road verge, $V_{t}$ is traffic speed, $L_{t}$ the length of rural principal A roads and rural minor roads with traffic at any one instant (second) and $L_{r}$ is the length of rural principal A roads and rural minor roads.

The probability that a mammal, once on a road, will be hit by a vehicle $\left(P_{h}\right)$ is given by equation 2 , where $T$ is the length of time taken by a mammal to cross a road.

$$
P_{h}=\frac{T L_{t}}{L_{r}}
$$

The probability of traffic-dependent mammal-vehicle collision $\left(P_{c h}\right)$ is the product of the two forgoing estimates. 


\section{Results}

Our dataset was derived from standardised journeys covering different landscape types and $10,202 \mathrm{~km}$ of roads driven (approximately $3 \%$ of the total length of all UK roads: $14 \%$ of the length of all 'A' roads, $11 \%$ of ' $\mathrm{B}$ ' roads and $0.4 \%$ of 'Minor' roads). In total 893 dead rabbits and 164 dead hedgehogs were counted.

Traffic flow was recorded as the count of all oncoming vehicles during a journey.

Both rabbit counts and traffic flow were related to road width (Figure 1).

\section{Rabbits counts, traffic flow and road width}

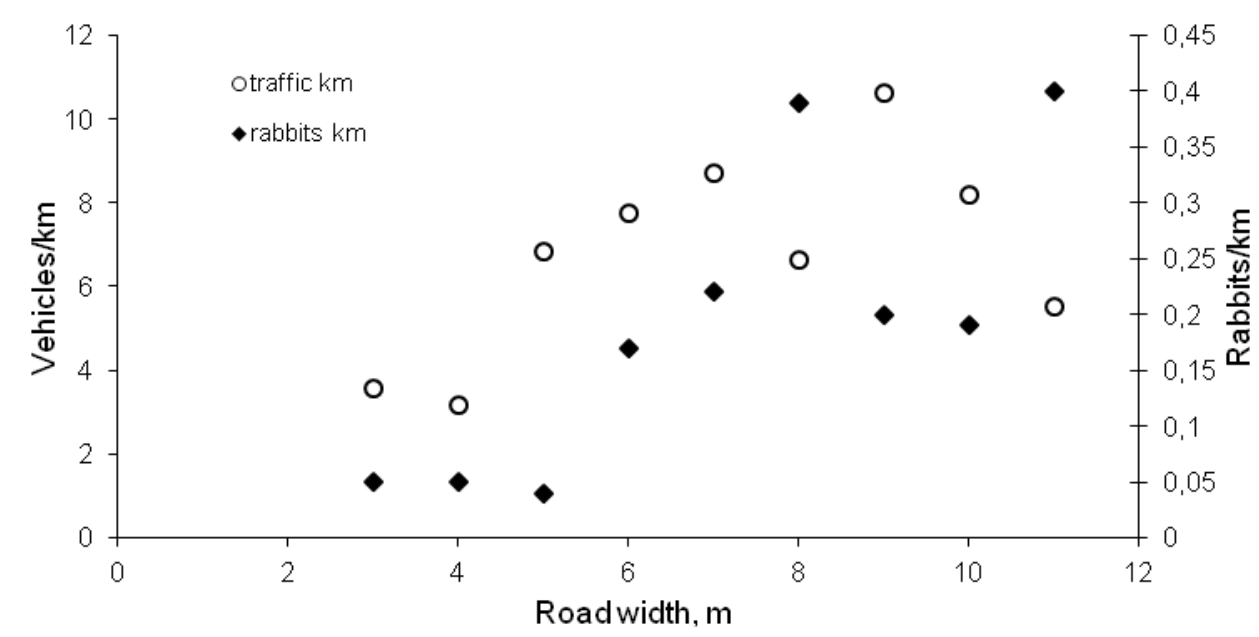

Figure 1. The relationships between road width, traffic flow and rabbit road counts.

However road width, but not traffic flow was a (strong) predictor of rabbit road kill counts $\left(\mathrm{F}=20.86\right.$, d.f. $\left.1,41, \mathrm{P}<0.001, \mathrm{r}^{2}=33 \%\right)$. Including the (non-significant) traffic flow term in the above model increased $\mathrm{r}^{2}$ by only $2 \%$. The situation was very similar for hedgehog (Figure 2).

Hedgehog counts, traffic flow and road width

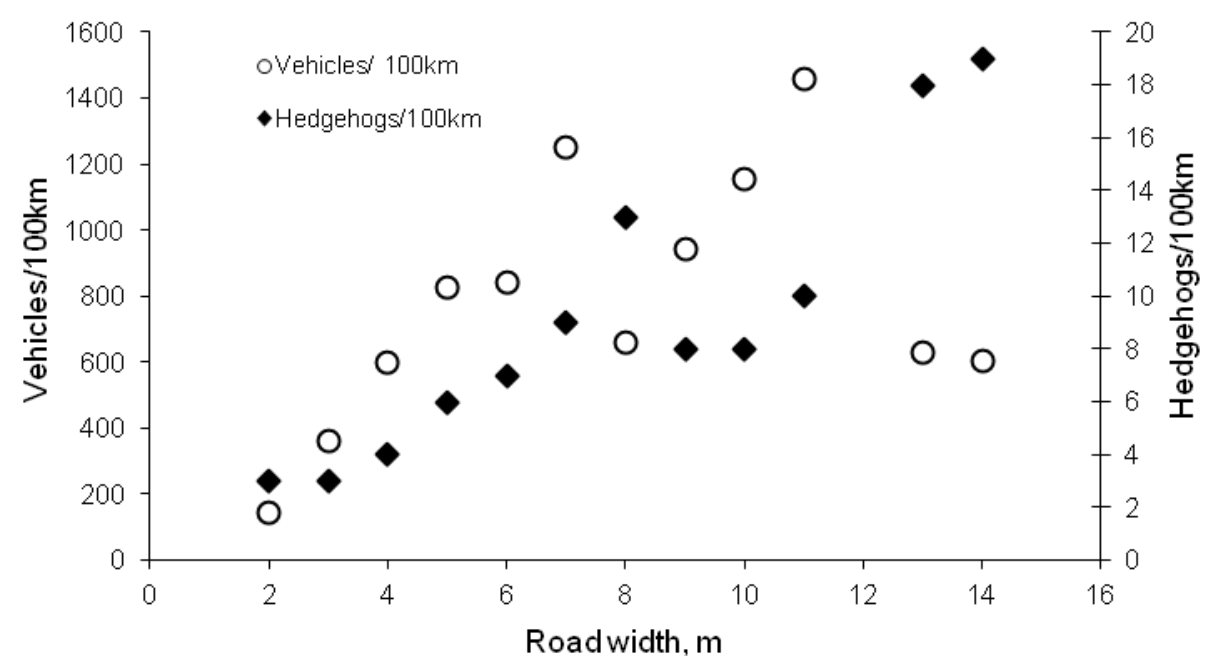

Figure 2. The relationships between road width, traffic flow and hedgehog road counts. 
Road width, but not traffic flow, was a strong predictor of hedgehog road kill counts $\left(\mathrm{F}=27.73\right.$, d.f. $\left.1,63, \mathrm{p}<0.001, \mathrm{r}^{2}=30 \%\right)$. Including the (non-significant) traffic flow term in the model did not increase $r^{2}$ at all. These data suggest that it is road width, not traffic flow that influences road kill counts. However, traffic flow and road width are, inevitably, autocorrelated and we cannot rule out the possibility that the effect of one masks that of the other.

It is highly instructive to consider how the increase in traffic flow has affected what we here term the 'safety window' i.e. the period of time when a section of road will be free from traffic and a mammal will be able to cross without risk of colliding with vehicles. We used 2004 traffic flow data (vehicle kilometres driven per year) for rural principal A roads and rural minor roads (Table 1.3 in Transport Statistics Bulletin 2004 (DTLR, 2005)). This we divided by the length of the same road types (Table 4.1b in Transport Statistics Bulletin 2004) to give a measure of the mean length of time between vehicles during day and night (the safety window). Then, using the ratio of day time to night time (here defined as 19:00 to 05:00) traffic flow (Table 3.3, Transport Statistics Bulletin 2004; day time traffic flow is 3.86 times that of night time traffic) we estimated the night time safety window.

During day and night a safety window of 10 seconds was available on $89 \%$ of the rural road network in Scotland and $76 \%$ and $66 \%$ of the network in England and South East England respectively (Figure 3).

$\%$ of roads with a given safety window for road crossing mammals, day \& night

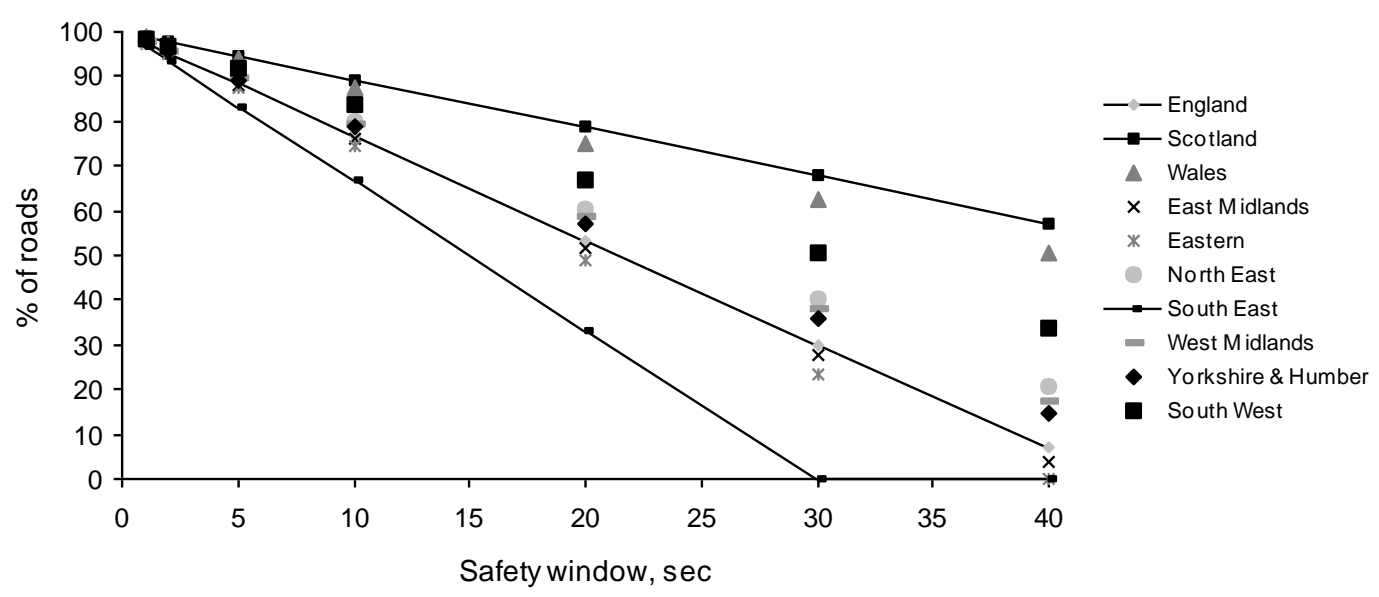

Figure 3. The percentage of the road network in rural areas without vehicular traffic for a given length of time (safety window, seconds). Data for day and night time. The lines, top to bottom, represent Scotland, England and South East England.

During the night a safety window of 10 seconds was available on $97 \%, 93 \%$ and 91\% of the road network in Scotland, England and South East England. A night time safety window of 40 seconds was available on $85 \%, 75 \%$ and $65 \%$ of the road network in the same regions (Figure 4). 
$\%$ of roads with a given safety window for road crossing mammals, night

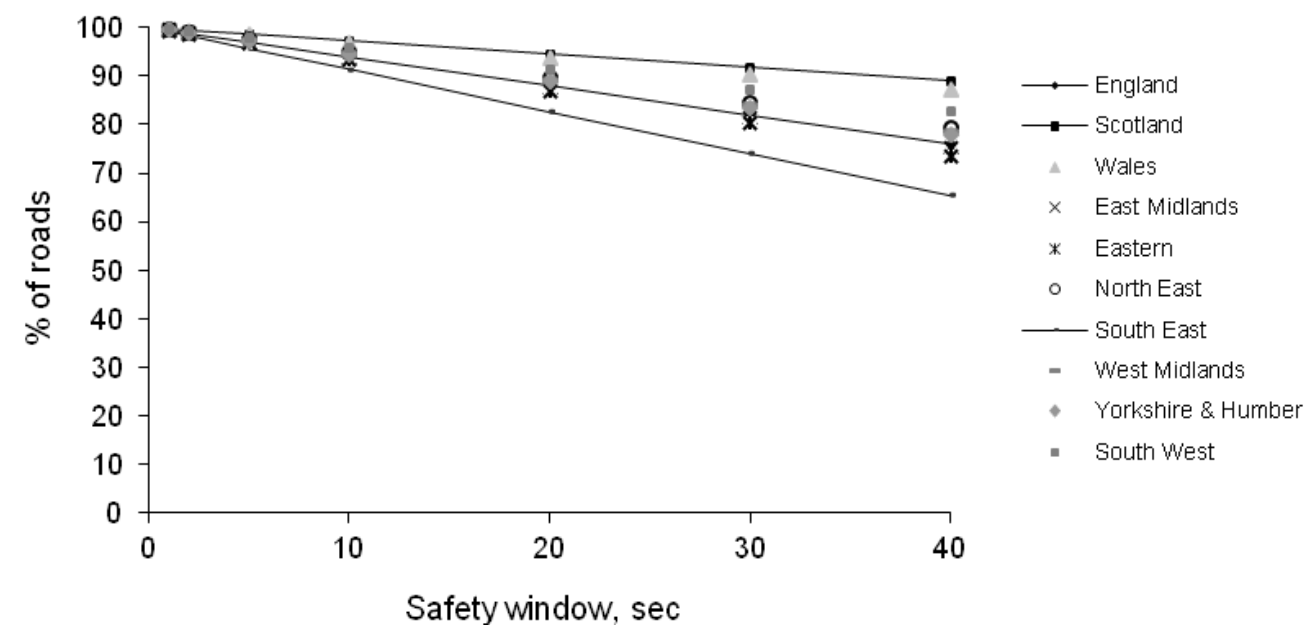

Figure 4. The percentage of the road network in rural areas without vehicular traffic for a given length of time (safety window, seconds). Data for night time only (19:00 to 05:00). The lines, top to bottom, represent Scotland, England and South East England.

These calculations suggest that most of the road network will offer ample time for hedgehogs and rabbits to cross even wide rural roads (time to cross a $10 \mathrm{~m}$ wide road: hedgehogs 20 seconds, rabbits 0.64 seconds).

It is clear that for both rabbits and hedgehogs the vast majority of the rural road network is likely to be perceived as free from risk of oncoming vehicles and the trafficdependent probability of road crossing is thus very high (Figure 5).

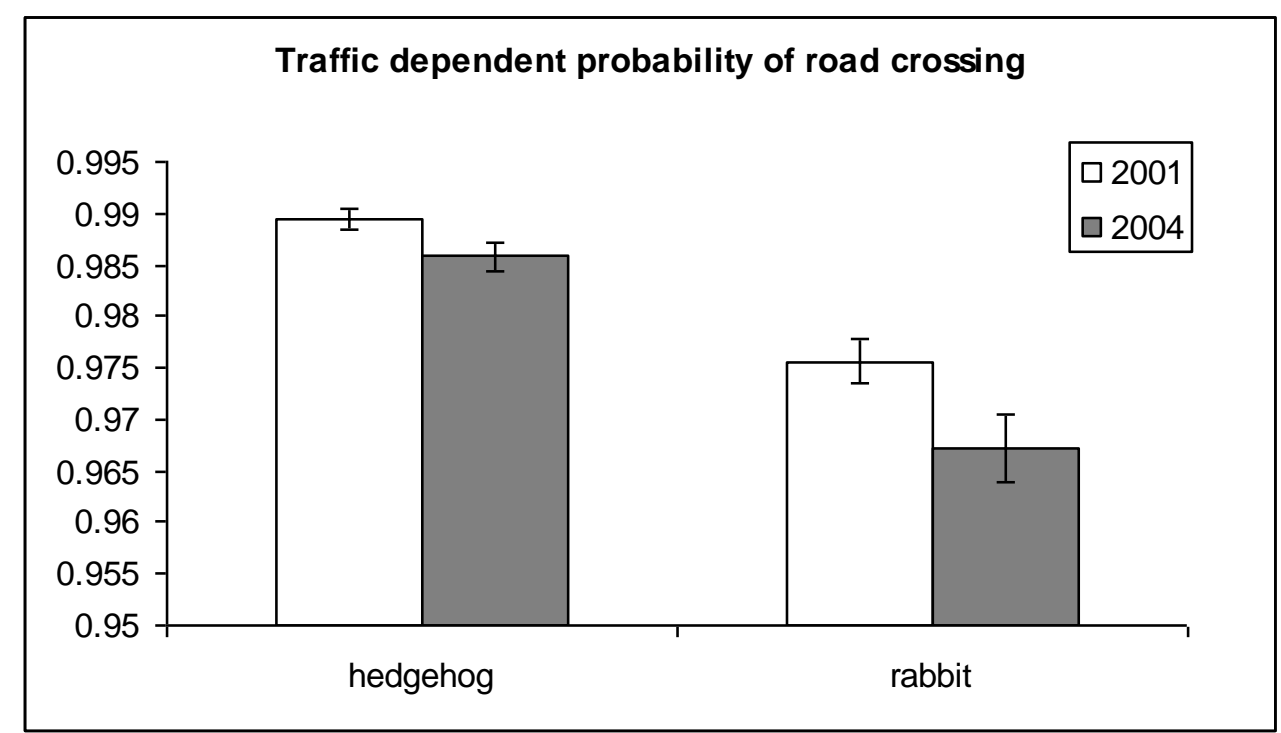

Figure 5. The influence of traffic flow on the probability of animals (hedgehog and rabbit) crossing roads (UK mean \pm s.e.). 
However because traffic speeds are high compared with speeds of mammal road crossing (hedgehogs $0.5 \mathrm{~m}^{-1} \mathrm{~s}^{-1}$; rabbits $15.5 \mathrm{~m}^{-1} \mathrm{~s}^{-1}$; vehicle travelling at $60 \mathrm{mph}$, $26.6 \mathrm{~m}^{-1} \mathrm{~s}^{-1}$ ), even risk-averse road-crossing mammals are at risk from collisions with vehicles.

The probability of rabbits being hit by a vehicle when on a road was, due to their much greater speed, two orders of magnitude less than that for hedgehogs (Figure 6).

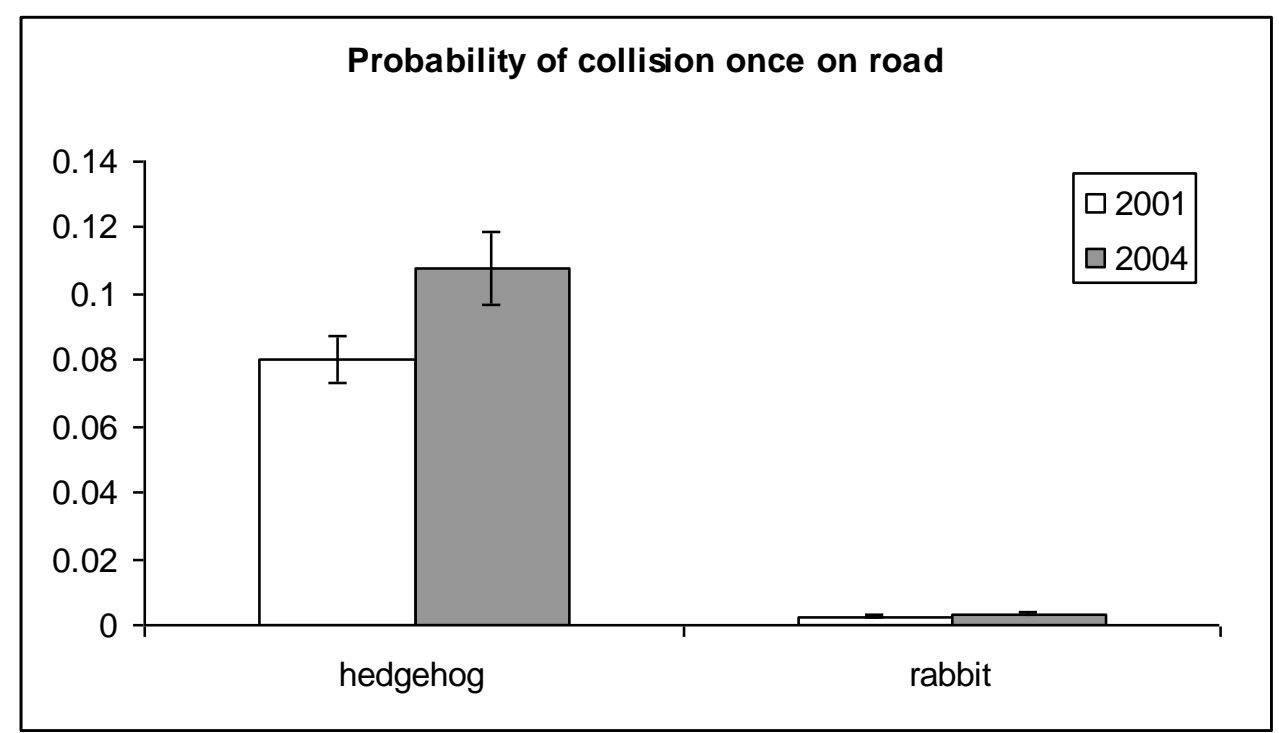

Figure 6. The influence of traffic flow on the probability of animals (hedgehog and rabbit) colliding with a vehicle once on road.

The overall traffic-dependent probability of rabbits colliding with vehicles was very low. It increased from 0.0025 in 2001 to only 0.0033 in 2004 . We suggest that the small order of magnitude of this effect would not have been likely to influence rabbit road counts - rabbits will generally be too fast and too risk-averse. For hedgehogs, the same measure increased from 0.079 in 2001 to 0.10 in 2004. This could, perhaps, have influenced hedgehog road counts.

In view of the foregoing findings it is not surprising that there is no correlation between changes in traffic flow and road counts of hedgehog or rabbit (2001 to 2004, counts by country and region, for hedgehog: Spearman correlation coefficient $0.4, n=10$, $\mathrm{p}=0.286$; for rabbit: Spearman correlation coefficient $-0.075, \mathrm{n}=9, \mathrm{p}=0.849$ ). Clearly this a fairly crude test of association and traffic data at a finer spatial scale (10-km grid squares) are desirable.

\section{Discussion}

With reference to the potential effects of increasing traffic flow on road-kill counts, our results suggest that increased risk aversion resulting from higher traffic flow per se will not have been nearly sufficient to account for the 2001 to 2004 reduction in hedgehog road kill counts in England reported from the annual Mammals on Roads survey (Mammals Trust UK, 2005).

This conclusion holds provided the primary cue of risk perceived by hedgehogs is a direct one: the sight and sound of oncoming vehicles. It is of course possible that 
hedgehogs perceive greater risk from roads with more traffic (irrespective of road width) by using indirect olfactory cues. However, levels of pollutants (potential cues) next to rural roads are not likely to have increased sufficiently in three years to provide a reliably discriminatory cue of higher traffic flow. Furthermore, roads, even without traffic, are likely to be a very noisy environment to the olfactory sense of even an insectivore. We conclude that hedgehogs are not likely to have become more inhibited from crossing roads during the survey period. It is suggested that further research is required into the behavioural responses of rabbits and hedgehogs (the two species most commonly seen dead on UK roads) to roads and traffic, with the aim of determining how these behavioural responses affect the probability of an individual animal crossing a road, and dying as it does so. This is relevant because it has important bearing on the relationship between population density and road-kill counts. For example, given a certain population density, road casualty numbers are likely to be higher for those species that respond to oncoming traffic by freezing in the road, compared to those whose members run for the verge.

The decrease in hedgehog road kill counts could be the direct or partial result of increased road traffic mortality reducing hedgehog abundance. Huijser (2000) found that traffic flow had no significant impact on hedgehog population density, though his study was only capable of detecting changes of more than $35 \%$ in hedgehog density. Clearly, however, if road mortality is directly affecting hedgehog abundance and hedgehog road counts are decreasing, we can be certain that hedgehog abundance is declining. If road mortality is affecting abundance then an increase in road counts might underestimate increases in absolute abundance. A decrease in road kill counts, however, will still be a good index of a decrease in absolute abundance.

It is crucial to understand what these calculations can and cannot show. They are used here only to explore the relations between changes in traffic flow and the likelihood of mammal road casualties. They do not provide absolute estimates of the probabilities of mammal road crossing or of mammal-vehicle collisions occurring. Rather, they show only how traffic flow may impact on these probabilities. Our calculations are based on very extensive traffic monitoring by the Department of Transport, but this is designed to show changes in traffic flow in the order of hundreds of thousands of vehicle-kilometres driven per year. It may thus not sort well with extrapolation to a scale of metres which is needed in understanding risks to mammals from road crossing. Nevertheless our estimates of the risks of road crossing are likely to be overestimates because traffic is clumped in space, not evenly distributed over the rural road network as our calculations assume and especially because we assume wide $(10 \mathrm{~m})$ roads.

In conclusion, our results suggest that a decrease in road counts of hedgehog, and in general, is highly likely to represent a decrease in absolute abundance (at a high rate, albeit over only four years) and thus be a good index with which to monitor population decline. Increases in road counts coincident with increases in traffic flow need to be viewed with more caution.

Acknowledgements. Thank you to the People's Trust for Endangered Species for supporting this study, and to Pat Morris for helpful discussions and for encouraging our interest in the issues of hedgehog road kill. 


\section{REFERENCES}

[1] Arnold, D.A. (1978): Characteristics and costs of highway deer kills. - In: Kirkpatrick C.M. (ed) Wildlife and People, John S. Wright Forestry Conf. Purdue Univ., West Lafayette, Indiana.

[2] Bellis, E.D., Graves, H.B. (1971): Deer mortality on a Pennsylvania Interstate. - Journal of Wildlife Management 35: 232-237.

[3] Brockie, R.E., Sadleir, R.M.F.S., Linklater, W.L. (2009): Long-term wildlife roadk-kill counts in New Zealand. - New Zealand Journal of Zoology 36(2): 123-134.

[4] Bunce, R.G.H., Barr, C.J., Gillespie, M.K., Howard, D.C. (1996): The ITE Land Classification: Providing an environmental stratification of Great Britain. Environmental Monitoring and Assessment 39: 39-46.

[5] Case, R.M. (1978): Interstate highway road killed animals: a data source for biologists. Wildlife Society Bulletin 6: 8-13.

[6] Davies, J.L. (1957): A hedgehog road mortality index. - Proceedings of the Zoological Society of London 128: 606-608.

[7] Driessen, M.M., Mallick, S.A., Hocking, G.J. (1996): Habitat of the eastern barred bandicoot, Perameles gunnii, in Tasmania: An analysis of road-kills. - Wildlife Research 23: 721-727.

[8] DTLR (2005): Transport Statistics Bulletin 2004. Department for Transport Local Government and the Regions, London.

[9] Fahrig, L., Pedlar, J.H., Pope, S.E., Taylor, P.D., Wegner, J.F. (1995): Effect of Road Traffic on Amphibian Density. - Biological Conservation 73: 177-182.

[10] Garland, T. (1983): The relation between maximal running speed and body mass in terrestrial mammals. - Journal of Zoology 199: 157-170.

[11] George, L.J. (2004): Use of road casualty data to monitor mammal abundance. PhD thesis, Royal Holloway University of London, Egham, Surrey.

[12] George, L.J., MacPherson, J.L., Balmforth, Z., Bright, P.W. (2011): Using the dead to monitor the living: can road kill counts detect trends in animal abundance? - Applied Ecology and Environmental Research 9(1): 27-41.

[13] Hicks, A.C. (1993): Using road-kills as an index to moose population change. - Alces 29: 243-247.

[14] Huijser, M.P. (2000): Life on the edge: hedgehog traffic victims and mitigation strategies in an anthropogenic landscape. PhD thesis, Wageningen University, Wageningen, the Netherlands.

[15] Inbar, M., Mayer, R.T. (1999): Spatio-temporal trends in armadillo diurnal activity and road kills in central Florida. - Wildlife Society Bulletin 27: 865-872.

[16] Jahn, L.R. (1959): Highway mortality as an index of deer population change. - Journal of Wildlife Management 23: 187-197.

[17] Mallick, S.A., Hocking, G.J., Driessen, M.M. (1998): Road-kills of the eastern barred bandicoot (Perameles gunnii) in Tasmania: an index of abundance. - Wildlife Research 25: 139-145.

[18] Mammals' Trust UK (2005) Mammals on Roads newsletter, hedgehogs p. 3

[19] McCaffery, K.R. (1973): Road kills show trends in Wisconsin deer populations. Journal of Wildlife Management 37(2): 212-216.

[20] Morris, P.A., Morris, M.M. (1988): Distribution and abundance of hedgehogs (Erinaceus europaeus) on New Zealand roads. - New Zealand Journal of Zoology 15: 491-498.

[21] Rolley, R.E., Lehman, L.E. (1992): Relationships among raccoon road-kill surveys, harvests and traffic. - Wildlife Society Bulletin 20: 313-318.

[22] Slater, F. (1994): Wildlife road casualties. - British Wildlife 5: 214-221. 